\title{
Confinement Stabilizes a Bacterial Suspension into a Spiral Vortex
}

\author{
Hugo Wioland, ${ }^{1}$ Francis G. Woodhouse, ${ }^{1}$ Jörn Dunkel, ${ }^{1}$ John O. Kessler, ${ }^{2}$ and Raymond E. Goldstein ${ }^{1}$ \\ ${ }^{1}$ Department of Applied Mathematics and Theoretical Physics, Centre for Mathematical Sciences, \\ University of Cambridge, Wilberforce Road, Cambridge CB3 OWA, United Kingdom \\ ${ }^{2}$ Department of Physics, University of Arizona, Tucson, Arizona 85721, USA
}

(Received 10 April 2013; published 24 June 2013)

\begin{abstract}
Confining surfaces play crucial roles in dynamics, transport, and order in many physical systems, but their effects on active matter, a broad class of dynamically self-organizing systems, are poorly understood. We investigate here the influence of global confinement and surface curvature on collective motion by studying the flow and orientational order within small droplets of a dense bacterial suspension. The competition between radial confinement, self-propulsion, steric interactions, and hydrodynamics robustly induces an intriguing steady single-vortex state, in which cells align in inward spiraling patterns accompanied by a thin counterrotating boundary layer. A minimal continuum model is shown to be in good agreement with these observations.
\end{abstract}

DOI: 10.1103/PhysRevLett.110.268102

PACS numbers: 87.18.Hf, 47.54.-r, 47.63.Gd, 87.17.Jj

Geometric boundaries and surface interactions are known to have profound effects on transport and order in condensed matter systems, with examples ranging from nanoscale edge currents in quantum Hall devices [1,2] to topological frustration in liquid crystals (LCs) tuned by manipulating molecular alignment at confining surfaces [3]. By contrast, in spite of considerable recent interest [4-8], the effects of external geometric constraints and confining interfaces on collective dynamics of active biological matter $[9,10]$, such as polar gels $[11,12]$ and bacterial [13-18] or algal suspensions [19], are not yet well understood, not least owing to a lack of well-controlled experimental systems.

At high concentrations, motile rodlike cells exhibit selforganization akin to nematic LC ordering [13,14,20], with the added facet of polar alignment driven by collective swimming [21,22]. Unlike passive LCs, cellular suspensions are in a constant state of flux: at scales between $10 \mu \mathrm{m}$ and $1 \mathrm{~mm}$, coherent structures (swirls, jets, and plumes) continually emerge and persist for seconds at a time $[14-17,23,24]$. While some progress has been made in understanding the dynamics of dense bacterial suspensions in bulk [16,18,23-26], microorganisms often live in porous habitats like soil, where encounters with interfaces or three-phase contact lines are common [13,14,27]. Recent work has clarified how single cells interact with surfaces [28-31], but it remains unclear how global geometric constraints influence their collective motion.

Here, we combine experiment and theory to investigate how confinement and boundary curvature affect stability

Published by the American Physical Society under the terms of the Creative Commons Attribution 3.0 License. Further distribution of this work must maintain attribution to the author(s) and the published article's title, journal citation, and DOI. and topology of collective dynamics in active suspensions. The physical system we study is an oil emulsion containing droplets of a highly concentrated aqueous suspension of Bacillus subtilis [Fig. 1(a)]. For drops of diameter $d=$ $30-70 \mu \mathrm{m}$ and height $h \sim 25 \mu \mathrm{m}$, we find that the suspension self-organizes into a single stable vortex [Fig. 1(b)] that persists as long as oxygen is available. This pattern is reminiscent of structures seen in colonies on the surface of agar [32], spontaneously circulating cytoplasmic extracts of algal cells [6], and the rotating interior of fibroblasts on micropatterned surfaces [33]. The vortex flow described here is purely azimuthal and accompanied by a thin counterrotating boundary layer, consisting of cells swimming opposite to the bulk. Surprisingly, we observe that the cells arrange in spirals with a maximum pitch angle of up to $35^{\circ}$ relative to the azimuthal bulk flow direction [Fig. 1(b)]. We suggest that this intriguing helical pattern results from the interplay of boundary curvature and steric and hydrodynamic interactions. Building on this hypothesis, we formulate a simple continuum model and find good agreement between its predictions and experimental results.
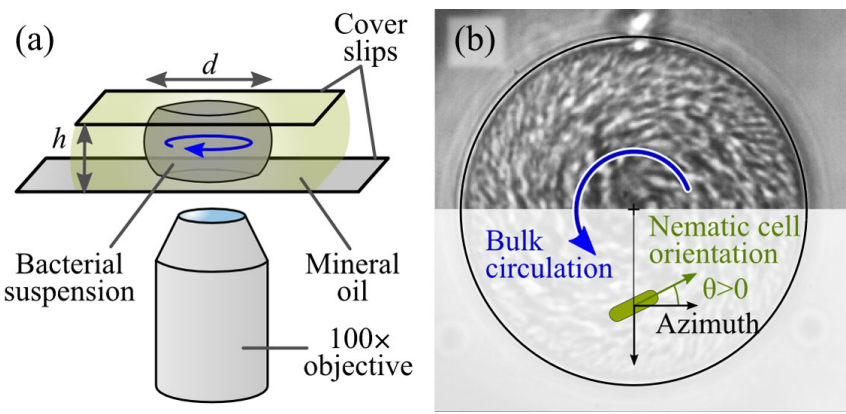

FIG. 1 (color online). Overview. (a) Experimental setup. (b) Bright field image of a $40 \mu \mathrm{m}$ drop, and definition of cell orientation angle relative to main circulation direction. 

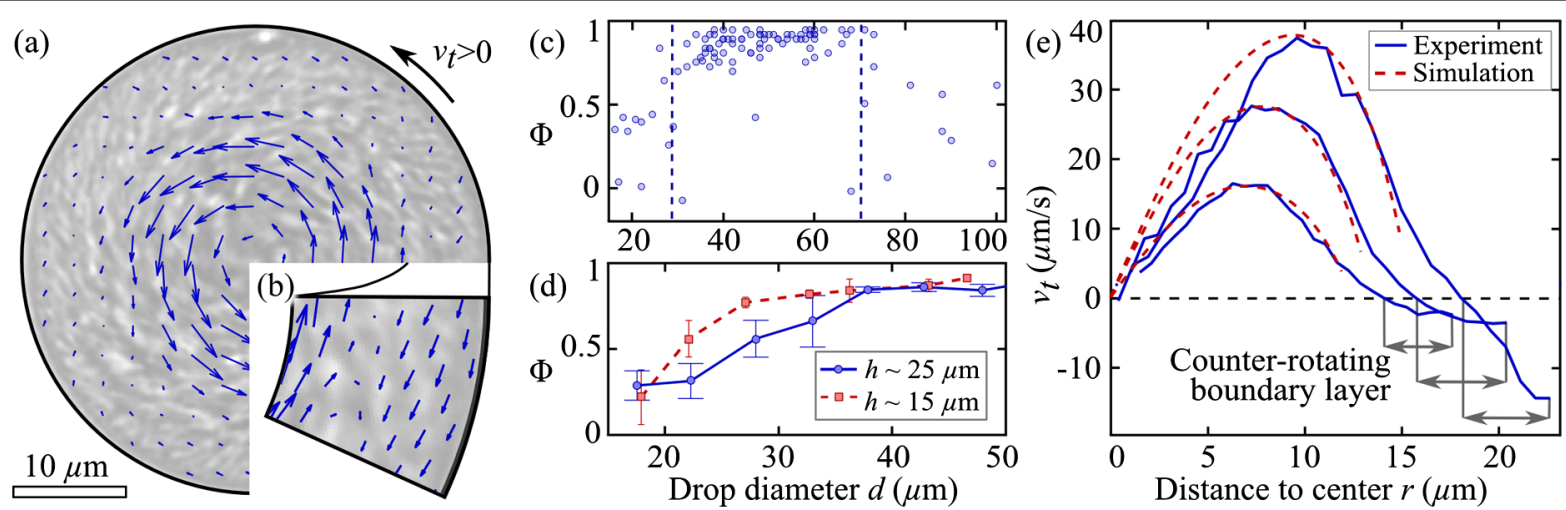

FIG. 2 (color online). Steady-state circulation in highly concentrated B. subtilis droplets. (a) PIV flow field for a droplet with a volume filling fraction $\varphi \sim 0.4$. For clarity, not all PIV vectors are shown. (b) Enlarged region reveals the counterrotating boundary layer. All PIV vectors are shown. (c),(d) Vortex order parameter $\Phi$ for varying diameter $d$. (c) Drops of constant height $h \sim 25 \mu \mathrm{m}$. Dashed lines denote the highly ordered single-vortex regime. (d) Averaged vortex order parameter $\Phi(5 \mu \mathrm{m}$ bins) for $h \sim 15 \mu \mathrm{m}$ (red dashed line) and $h \sim 25 \mu \mathrm{m}$ (blue solid line). Error bars indicate the standard deviation. (e) Azimuthal flow $v_{t}(r)=\langle\mathbf{v} \cdot \mathbf{t}\rangle_{\theta}$ profile for three different experiments (blue solid lines), compared with continuum bulk flow model results (red dashed lines). Negative flow indicates the counterrotating boundary layer.

B. subtilis (wild-type strain 168) were grown in standard Terrific Broth (TB, Sigma) at $35^{\circ} \mathrm{C}$ on a shaker. An overnight culture was diluted $200 \times$ and grown for $5 \mathrm{~h}$ until the end of exponential growth when the proportion of motile cells is maximal [34]. Cells were then centrifuged at $1500 \mathrm{~g}$ for $10 \mathrm{~min}$. The pellet was gently mixed and transferred to 4 volumes of mineral oil, with $10 \mathrm{mg} / \mathrm{mL}$ diphytanoyl phosphatidylcholine (DiPhyPC, Avanti) added to prevent the emulsion from coalescing. Small drops were created by slowly pipetting the suspension, $10 \mu \mathrm{L}$ of which was placed between two coverslips such that it spread by surface tension to the coverslip edge. This procedure yielded many flattened drops with $h \sim 25 \mu \mathrm{m}$ and diameters ranging from $10-150 \mu \mathrm{m}$, and bacterial volume fraction $\varphi \sim 0.4$. The surrounding oil provided a source of oxygen necessary for bacterial motion. The relatively smaller diffusive influx in the largest drops resulted in an oxygen gradient that we avoided by taking movies in the very first minutes of the experiment, while bacteria in smaller drops would swim in a steady state vortex for more than $10 \mathrm{~min}$. Coverslips were rendered hydrophobic with silane, resulting in pancake-shaped drops that are wider at the midplane of the chamber than at the top and bottom [Fig. 1(a)]. Movies were acquired at 125 fps with a highspeed camera (Fastcam, Photron) on an inverted microscope (Cell Observer, Zeiss), using a $100 \times$ oil-immersion objective and analyzed with custom Matlab algorithms. Flows were imaged in the center of the chamber to minimize optical distortions.

Confinement by the oil interface stabilizes rapidly rotating vortices (Fig. 2 and Video 1 in the Supplemental Material [35]). To quantify this effect, we determined the local bacterial velocity field $\mathbf{v}(\mathbf{x})$, using a customized version of the particle image velocimetry (PIV) toolbox mPIV [36] that averages pixel correlations over two seconds [37]. The PIV algorithm yields the local mean velocity of the bacteria, reflecting locomotion due to swimming and advection by flow [Fig. 2(a)]. The emergence of stable azimuthal flow is captured by the vortex order parameter

$$
\Phi=\frac{\sum_{i}\left|\mathbf{v}_{i} \cdot \mathbf{t}_{i}\right| / \sum_{j}\left\|\mathbf{v}_{j}\right\|-2 / \pi}{1-2 / \pi},
$$

where $\mathbf{v}_{i}$ is the in-plane velocity and $\mathbf{t}_{i}$ the azimuthal unit vector [Fig. 1(b)] at PIV grid point $\mathbf{x}_{i} \cdot \Phi=1$ for steady azimuthal circulation, $\Phi=0$ for disordered chaotic flows, and $\Phi<0$ for predominantly radial flows. Plotting $\Phi$ as a function of drop diameter reveals that a highly ordered single-vortex state with $\Phi>0.7$ forms if $d_{-}<d<d_{+}$ with $d_{-} \sim 30 \mu \mathrm{m}$ and $d_{+} \sim 70 \mu \mathrm{m}$ [Fig. 2(c)]. Clockwise and counterclockwise vortices occur with equal probability. The lower critical diameter $d_{-}$depends on the chamber height $h$ [Fig. 2(d)]. Lowering $h$ restores the quasi-2D nature of the confinement and allows for formation of vortex states at smaller diameter $d$. The upper critical diameter $d_{+}$is consistent with the size of the transient turbulent swirls observed in 3D bulk bacterial suspensions $[16,18,24]$. In drops slightly larger than $d_{+}$flow is still azimuthal near the boundary regions but the vortex order decreases toward the center. Drops with $d \geqslant 100 \mu \mathrm{m}$ show fully developed bacterial turbulence as seen in quasi-infinite suspensions $[14,16,18,24]$.

The azimuthal flow speed in a vortex state is maximal at a distance $\sim d / 4$ from the center [Fig. 2(e)]. Across experiments, the maximum speed increases with $d$, reaching $\sim 40 \mu \mathrm{m} / \mathrm{s}$ for $d_{+}$, roughly four times the typical swimming speed of an isolated bacterium [17] and in agreement with measurements in open $B$. subtilis suspensions $[16,17]$. While our setup does not supply oxygen, and the bacterial motility decreases [18] with time, recent 
studies of quasi-infinite suspensions [18,24] have shown that the flow correlation length is independent of swimming speed at high cell density, so we may neglect oxygen depletion in the analysis of patterns. In the following, we focus on the properties of single-vortex states with $\Phi>0.7$ and take the azimuthal unit vector $\mathbf{t}$ to point in the direction of bulk flow, so that we may treat clockwise and counterclockwise vortices equally [Fig. 1(b)].

Detailed flow field analysis reveals that highly ordered vortex states are always accompanied by a thin layer of cells swimming along the oil interface in the opposite direction to the bulk flow [Fig. 2(b)]. This surprising fact is reflected in the azimuthally averaged velocity profile $v_{t}(r)=\langle\mathbf{v}(\mathbf{x}) \cdot \mathbf{t}\rangle_{\theta}$, where $\mathbf{x}=(r \cos \theta, r \sin \theta)$, which changes sign near the edge of the droplet [Fig. 2(e)]. The basic form of $v_{t}(r)$ is preserved among all well-ordered droplets with $\Phi>0.7$ [Fig. 2(e)]. To exclude the possibility that the backflow arises from interactions between bacteria, DiPhyPC and oil, we performed control experiments with dense suspensions in shallow cylindrical polydimethylsiloxane chambers, and found similar behavior. This suggests that the formation of a thin counterflow boundary layer is a generic phenomenon in bacterial suspensions confined by a higher-viscosity medium (Video 2 in Supplemental Material [35]). By determining the zeros of $v_{t}$ for all ordered droplets, we find that the boundary layer thickness $b$ is independent of $d$ [Fig. 2(e)]. The average value $\bar{b} \approx 4 \mu \mathrm{m}$ is slightly smaller than the length $\ell \approx 5 \mu \mathrm{m}$ of $B$. subtilis [23], suggesting that the counterflow is composed of a single layer of cells. By imaging droplets in a plane near the bottom cover slip in order to resolve vertical cell layers more easily, we confirmed that cells swimming in the direction opposite to the bulk flow are in direct contact with the oil interface [Fig. 3(a) and Video 3 in the Supplemental Material [35]].

The presence of this previously unreported counterflow layer can be understood by considering the main forces causing reorientation near the boundary. Since the oil
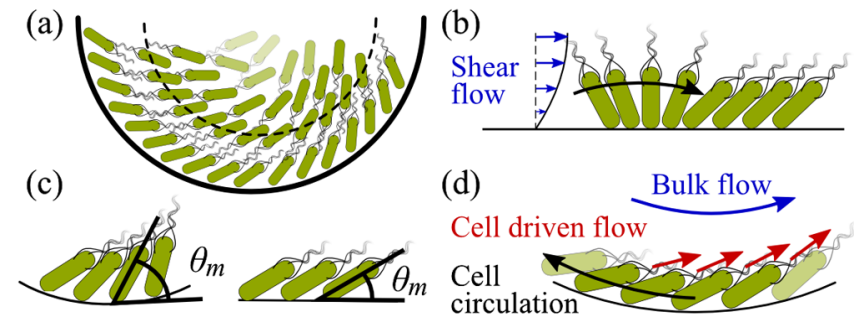

FIG. 3 (color online). Schematic cell organization in droplets. (a) Dashed line indicates continuum model boundary, where bulk flow begins. (b)-(d) Physical mechanisms driving boundary layer formation. (b) Shear flow reorients cells to face upstream. (c) Contact angle $\theta_{m}$ decreases with the drop diameter, restricted by steric interactions. (d) Ratchetlike steric repulsion and inward flow (red arrows) created by boundary cells force the next layer to move in the opposite azimuthal direction, thereby setting the bulk flow direction. viscosity is $10 \times$ that of water, the interface acts as a nearly no-slip boundary. Thus, circular bulk motion creates a shear flow that exerts torque on the cells in the boundary layer [Fig. 3(b)]. As recently shown for dilute suspensions [38], bacteria prefer to swim upstream when exposed to such flow gradients, thereby favoring the formation of a counterrotating layer. If the concentration of cells is sufficiently high, nematic ordering due to steric interactions further stabilizes this layer $[10,21,22]$. Once the layer has formed, cells trapped in it form a ratchetlike structure and, because they are pusher-type swimmers [31], they generate a backflow in the direction opposite to their orientation [Fig. 3(d)]. Both effects force cells in the second layer to move in the other direction: the boundary monolayer stabilizes the bulk flow and vice versa. The absence of such backflow in the free-boundary geometry of Czirok et al. [32] provides further evidence that this is a consequence of rigid boundary effects.

A dense suspension of rodlike bacteria locally aligns through active nematic interactions [10,21,22]. We observe cell orientation that is not parallel to the flow direction: in the bulk circulation the cells point inwards, and in the boundary layer they point outwards [Fig. 3(a)]. We extract the local mean orientation from the bacterial speckle by computing the orientation tensor [35,39] [Fig. 4(a)]. As for the flow, we examine the azimuthally averaged orientation angle $\theta(r)$ relative to the circulation direction $\mathbf{t}$. Near the center of a drop, cells are aligned roughly parallel to the bulk circulation $(\theta \approx 0)$, and the angle increases with $r$ to a maximum value $\theta_{m}$ close to the boundary. Viewing $\theta_{m}$ as a function of $d$, we find an inverse correlation: the smaller the drop (and thus the higher the boundary curvature), the larger the deviation from the azimuthal direction, ranging from $\theta_{m} \sim 10^{\circ}$ for $d=70 \mu \mathrm{m}$ to $\theta_{m} \sim 35^{\circ}$ for $d=30 \mu \mathrm{m}$ [Fig. 4(b)]. To test whether $\theta_{m}$ depends on the curvature or on the suspension size, we performed measurements with suspensions diluted to $\sim 2 / 3$ of the starting concentration.
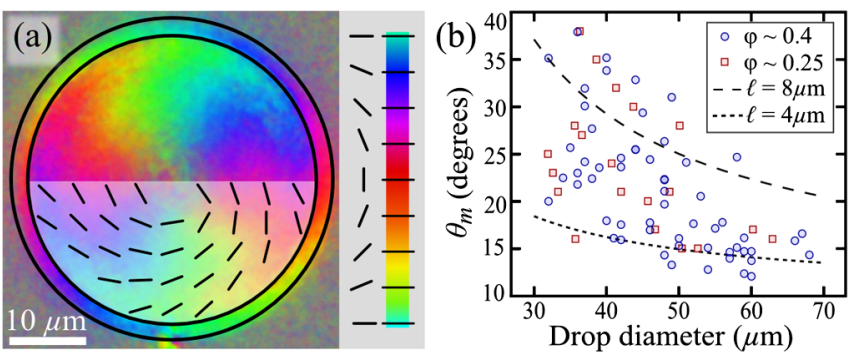

FIG. 4 (color online). Bacterial orientation. (a) Local orientation, averaged over $2 \mathrm{~s}$. External ring lies at the water-oil interface and shows local azimuthal direction $\mathbf{t}$, and cellular orientation appears in the central disc. Discontinuity in color between ring and disc indicates the angle between cells and the azimuthal direction. (b) Boundary angle [Fig. 3(c)] as a function of drop diameter. Symbols denote different bacterial concentrations. Dashed black lines indicate geometric estimates of minimum packing angle $\Theta$ for different cell lengths. 
In such drops, cells concentrate at the boundary, leaving the center almost empty (Video 4 in the Supplemental Material [35]). Yet, the measured angles are comparable to those of fully concentrated suspensions [Fig. 4(b)], indicating that this is indeed an effect of boundary curvature.

To explain this phenomenon, we consider purely steric packing in the boundary layer. This viewpoint is supported by the simulations of Wensink and Löwen [5] which show that a group of self-propelled particles does not align parallel to a boundary but instead lies at an angle limited by steric repulsion. Given a bacterial concentration, we model cells as thin rectangles equally spaced around a circle of diameter $d$ and calculate the minimum angle $\Theta$ with the azimuthal direction at which the cells could lie in one plane. A dilute suspension thus has edge-parallel packing $\left(\Theta=0^{\circ}\right)$, while at some limiting concentration they become boundary perpendicular $\left(\Theta=90^{\circ}\right)$. In the intermediate regime, $\Theta$ decreases as drop diameter $d$ increases [i.e., as boundary curvature falls; Fig. 3(c)]. Figure 4(b) illustrates packing curves for two cell lengths, $\ell=4 \mu \mathrm{m}$ and $\ell=8 \mu \mathrm{m}$, at a volume fraction of 0.5 . Measured values of $\theta_{m}$ lie between these curves, suggesting the scatter can be explained by variations in cell length (which was observed across experiments).

Recent work has demonstrated accurate modeling of dense bacterial flow in unbounded domains using phenomenological single-field models [23-25]. However, in this work we have been able to measure a second observable beyond the aggregate flow field-namely, the underlying bacterial orientation-and so a two-field model is necessary in order to fully reproduce the experimental results. Thus, as is typical in active suspension theory [40], we describe the system using two fields: the bacterial polar order parameter $\mathbf{P}$, where $|\mathbf{P}|=0$ for total disorder and $|\mathbf{P}|=1$ for total order with mean orientation direction $\mathbf{P}$, and the flow field $\mathbf{u}$ of the suspending fluid.

The flow is taken to be incompressible $(\nabla \cdot \mathbf{u}=0)$ and to obey the forced Stokes equations with friction:

$$
-\mu \nabla^{2} \mathbf{u}+\nu \mathbf{u}+\nabla \Pi=-c_{0} \sigma \nabla \cdot(\mathbf{P P}) .
$$

The frictional term $\nu \mathbf{u}$ captures the effects of vertical confinement (cf. Hele-Shaw flow) and high bacterial density (cf. Darcy's law), in addition to the usual viscous term with viscosity $\mu$. The flow, with pressure $\Pi$, responds to dipolar "pusher" forcing on the right-hand side of strength $\sigma$ for a suspension of concentration (number density) $c_{0}$.

To model the action of dense bacterial swimming, we define the swimming field $\mathbf{s}=V \mathbf{P}_{I}$, where $\mathbf{P}_{I}$ is the incompressible part of the bacterial orientation $\mathbf{P}$ (defined uniquely under Helmholtz decomposition) and $V$ is the bacterial swimming speed; this models swimming in a dense suspension where concentration fluctuations are negligible. The polar order $\mathbf{P}$ is then taken to evolve as

$$
\begin{aligned}
\partial_{t} \mathbf{P}+(\mathbf{u}+\mathbf{s}) \cdot \nabla \mathbf{P}= & D_{s} \nabla^{2} \mathbf{P}-D_{r} \mathbf{P}+\alpha\left(1-|\mathbf{P}|^{2}\right) \mathbf{P} \\
& +\epsilon(\mathbb{\square}-\mathbf{P P}) \cdot(\gamma \mathbf{E}+\mathbf{W}) \cdot \mathbf{P} .
\end{aligned}
$$

On the left-hand side, cells are advected by an aggregate flow field $\mathbf{u}+\mathbf{s}$. On the right-hand side, the terms are, in order, spatial and rotational diffusion with respective constants $D_{s}$ and $D_{r}$, spontaneous polar ordering of strength $\alpha$, and nematic reorientation induced by solvent strain $\mathbf{E}=\left(\nabla^{T} \mathbf{u}+\nabla \mathbf{u}^{T}\right) / 2$ and vorticity $\mathbf{W}=\left(\nabla^{T} \mathbf{u}-\nabla \mathbf{u}^{T}\right) / 2$, with cell shape parameter $\gamma \in[-1,1]$ and effectiveness $\epsilon \leq 1$ to incorporate inhibited reorientational freedom due to steric effects.

Finally, we must impose boundary conditions. These are provided by the bacterial boundary layer, which we have seen is a single-cell effect rather than part of the main suspension dynamics. This drives conditions at $r=d_{0} / 2$ of fixed orientation angle $\theta_{b}$; i.e., $\mathbf{P}=\mathbf{t} \cos \theta_{b}-\mathbf{r} \sin \theta_{b}$, where $\mathbf{r}$ is the outward radial unit vector. A no-slip boundary condition is imposed on the flow.

We seek to reproduce the experimental results shown in Fig. 2(e). To model the steady vortex regime we reduce to axisymmetry, where $\mathbf{u}=u \mathbf{t}$ and $\mathbf{s}=V(\mathbf{P} \cdot \mathbf{t}) \mathbf{t}$ by incompressibility, immediately yielding purely azimuthal flow as observed. Properties of B. subtilis [31] and of the suspending medium supply parameter values $\mu=$ $10^{-3} \mathrm{Pas}, D_{s}=10^{3} \mu \mathrm{m}^{2} \mathrm{~s}^{-1}, D_{r}=0.057 \mathrm{~s}^{-1}, \gamma=0.9$, and $\theta_{b}=20^{\circ}$, and the suspension volume fractions used here imply an approximate number density of $c_{0}=$ $0.1 \mu \mathrm{m}^{-3}$. Finally, we fix values for the remaining parameters $\epsilon=0.5, \alpha=25 \mathrm{~s}^{-1}$, and $\nu=10^{-4} \mathrm{Pas} \mu \mathrm{m}^{-2}$, which yield appropriate solutions for both the flow field and bacterial orientation $\theta(r)$ across different domain sizes. We simulated the three bulk domain diameters $d_{0}=24$, 26, $30 \mu \mathrm{m}$, and for each we set $V=4,7,10 \mu \mathrm{m} \mathrm{s}^{-1}$ and $\sigma=0.3,0.525,0.75 \mathrm{pN} \mu \mathrm{m}$, respectively; the dipole strength of a bacterium is known [31], and the scaling (holding $V / \sigma$ constant) reflects varying oxygen availability between experiments. These yield the steady-state curves of the lab frame bacterial flow $|\mathbf{u}+\mathbf{s}|$ in Fig. 2(e), exhibiting good agreement in the bulk. As shown in the Supplemental Material [35], the orientation angle $\theta(r)$ falls from its initial value $\theta\left(d_{0} / 2\right)=\theta_{b}$ as $r$ decreases, as observed.

The overall bacterial arrangement we have observed is reminiscent of rotating spirals [12] and striped phases [41] predicted for totally ordered active gels, although these models describe the actin-myosin cytoskeleton and lack interactions particular to microswimmer suspensions [42]. A more appropriate representation could be derived from polar active liquid crystals: the bacterial boundary layer could be regarded as a smectic structure [43] while the bulk behaves as a chiral nematic phase [3]. Yet, it is only by considering the microscopic hydrodynamics near the oil interface that the presence of the backflow layer can be inferred. This lends a note of caution to continuum 
modeling of microswimmer suspensions, suggesting that conditions at boundaries, and microscopic effects in general, warrant careful and deliberate consideration. Our combined experimental and theoretical results demonstrate that suitably designed boundaries provide a means for stabilizing and controlling order in active microbial systems.

We thank A. Honerkamp-Smith, V. Kantsler, P. Khuc Trong, K. Leptos, and E. Lushi for discussions. This work was supported by the EPSRC and ERC Advanced Investigator Grant No. 247333.

[1] K. von Klitzing, Physica (Amsterdam) 184B, 1 (1993).

[2] A. H. MacDonald and P. Středa, Phys. Rev. B 29, 1616 (1984).

[3] D. Seč, T. Porenta, M. Ravnik, and S. Žumer, Soft Matter 8, 11982 (2012).

[4] D. Grossman, I. S. Aranson, and E. B. Jacob, New J. Phys. 10, 023036 (2008).

[5] H. H. Wensink and H. Löwen, Phys. Rev. E 78, 031409 (2008).

[6] F. G. Woodhouse and R.E. Goldstein, Phys. Rev. Lett. 109, 168105 (2012).

[7] M. Ravnik and J. M. Yeomans, Phys. Rev. Lett. 110, 026001 (2013).

[8] T. Brotto, J.-B. Caussin, E. Lauga, and D. Bartolo, Phys. Rev. Lett. 110, 038101 (2013).

[9] D. L. Koch and G. Subramanian, Annu. Rev. Fluid Mech. 43, 637 (2011).

[10] T. Vicsek, A. Cziròk, E. Ben-Jacob, I. Cohen, and O. Shochet, Phys. Rev. Lett. 75, 1226 (1995).

[11] S. Fürthauer, M. Neef, S. W. Grill, K. Kruse, and F. Jülicher, New J. Phys. 14, 023001 (2012).

[12] K. Kruse, J. F. Joanny, F. Jülicher, J. Prost, and K. Sekimoto, Phys. Rev. Lett. 92, 078101 (2004).

[13] J.O. Kessler and M.F. Wojciechowski, in Bacteria as Multicellular Organisms, edited by J. A. Shapiro and M. Dworkin (Oxford University Press, New York, 1997), p. 417.

[14] C. Dombrowski, L. Cisneros, S. Chatkaew, R. E. Goldstein, and J. O. Kessler, Phys. Rev. Lett. 93, 098103 (2004).

[15] I. Tuval, L. Cisneros, C. Dombrowski, C. W. Wolgemuth, J. O. Kessler, and R. E. Goldstein, Proc. Natl. Acad. Sci. U.S.A. 102, 2277 (2005).

[16] L.H. Cisneros, R. Cortez, C. Dombrowski, R.E. Goldstein, and J. O. Kessler, Exp. Fluids 43, 737 (2007).

[17] A. Sokolov, I.S. Aranson, J. O. Kessler, and R.E. Goldstein, Phys. Rev. Lett. 98, 158102 (2007).

[18] A. Sokolov and I. S. Aranson, Phys. Rev. Lett. 109, 248109 (2012).

[19] S. Rafai, L. Jibuti, and P. Peyla, Phys. Rev. Lett. 104, 098102 (2010).
[20] D. Volfson, S. Cookson, J. Hasty, and L. S. Tsimring, Proc. Natl. Acad. Sci. U.S.A. 105, 15346 (2008).

[21] F. Ginelli, F. Peruani, M. Bär, and H. Chaté, Phys. Rev. Lett. 104, 184502 (2010).

[22] H. H. Wensink and H. Löwen, J. Phys. Condens. Matter 24, 464130 (2012).

[23] H. H. Wensink, J. Dunkel, S. Heidenreich, K. Drescher, R. E. Goldstein, H. Löwen, and J. M. Yeomans, Proc. Natl. Acad. Sci. U.S.A. 109, 14308 (2012).

[24] J. Dunkel, S. Heidenreich, K. Drescher, H. H. Wensink, M. Bär, and R. E. Goldstein, Phys. Rev. Lett. 110, 228102 (2013).

[25] J. Dunkel, S. Heidenreich, M. Bär, and R. E. Goldstein, New J. Phys. 15, 045016 (2013).

[26] E. Lushi and C.S. Peskin, Comput. Struct. 122, 239 (2013).

[27] W. M. Durham, O. Tranzer, A. Leombruni, and R. Stocker, Phys. Fluids 24, 091107 (2012).

[28] W. R. DiLuzio, L. Turner, M. Mayer, P. Garstecki, D. B. Weibel, H.C. Berg, and G. M. Whitesides, Nature (London) 435, 1271 (2005).

[29] G. Li, L.-K. Tam, and J. X. Tang, Proc. Natl. Acad. Sci. U.S.A. 105, 18355 (2008).

[30] V. Kantsler, J. Dunkel, M. Polin, and R. E. Goldstein, Proc. Natl. Acad. Sci. U.S.A. 110, 1187 (2013).

[31] K. Drescher, J. Dunkel, L. H. Cisneros, S. Ganguly, and R. E. Goldstein, Proc. Natl. Acad. Sci. U.S.A. 108, 10940 (2011).

[32] A. Czirók, E. Ben-Jacob, I. Cohen, and T. Vicsek, Phys. Rev. E 54, 1791 (1996).

[33] A. Kumar, A. Maitra, M. Sumit, G. V. Shivashankar, and S. Ramaswamy, arXiv:1302.6052.

[34] D. B. Kearns and R. Losick, Genes Dev. 19, 3083 (2005).

[35] See Supplemental Material at http://link.aps.org/ supplemental/10.1103/PhysRevLett.110.268102 for supplementary information and experimental movies.

[36] N. Mori and K.-A. Chang, www.oceanwave.jp/softwares/ mpiv/index.php?FrontPage (2006).

[37] C. D. Meinhart, S. T. Wereley, and J. G. Santiago, J. Fluids Eng. 122, 285 (2000).

[38] J. Hill, O. Kalkanci, J. L. McMurry, and H. Koser, Phys. Rev. Lett. 98, 068101 (2007).

[39] R. Rezakhaniha, A. Agianniotis, J.T.C. Schrauwen, A. Griffa, D. Sage, C. V. C. Bouten, F. N. Vosse, M. Unser, and N. Stergiopulos, Biomech. Model. Mechanobiol. 11, 461 (2012).

[40] D. Saintillan and M. J. Shelley, Phys. Rev. Lett. 100, 178103 (2008).

[41] R. Voituriez, J. F. Joanny, and J. Prost, Europhys. Lett. 70, 404 (2005).

[42] J.-F. Joanny and J. Prost, HFSP J. 3, 94 (2009).

[43] L. Giomi, M. C. Marchetti, and T. B. Liverpool, Phys. Rev. Lett. 101, 198101 (2008). 